\title{
Risk factors associated with adverse reactions to antituberculosis drugs*
}

\author{
Fatores de risco associados às reações \\ adversas a medicamentos antituberculose
}

\author{
Laíse Soares Oliveira Resende, Edson Theodoro dos Santos-Neto
}

\begin{abstract}
This review sought to identify the available scientific evidence on risk factors associated with adverse reactions to antituberculosis drugs. We performed a systematic review of studies published in the 1965-2012 period and indexed in the MEDLINE and LILACS databases. A total of 1,389 articles were initially selected. After reading their abstracts, we selected 85 studies. Of those 85 studies, 16 were included in the review. Risk factors for adverse reactions to antituberculosis drugs included age $>60$ years, treatment regimens, alcoholism, anemia, and HIV co-infection, as well as sodium, iron, and albumin deficiency. Protective factors against hepatic adverse effects of antituberculosis drugs included being male (combined OR $=0.38 ; 95 \% \mathrm{Cl}: 0.20-0.72$ ) and showing a rapid/intermediate $\mathrm{N}$-acetyltransferase 2 acetylator phenotype (combined $\mathrm{OR}=0.41 ; 95 \% \mathrm{Cl}$ : 0.18-0.90). There is evidence to support the need for management of adverse reactions to antituberculosis drugs at public health care facilities.
\end{abstract}

Keywords: Tuberculosis; Drug-related side effects and adverse reactions; Antitubercular agents; Review.

\section{Resumo}

Esta revisão buscou identificar a evidência científica disponível sobre os fatores de risco associados às reações adversas a medicamentos (RAM) antituberculose. Foi realizada uma revisão sistemática de estudos publicados entre 1965 a 2012 e indexados nas bases de dados MEDLINE e LILACS. Foram inicialmente selecionados 1.389 artigos. Após a leitura dos resumos, foram selecionados 85 estudos. Dos 85 estudos, 16 foram incluídos na revisão. Os fatores de risco de RAM foram idade > 60 anos, esquemas de tratamento, alcoolismo, anemia, coinfecção pelo HIV e deficiência de sódio, ferro e albumina. Os fatores de proteção contra RAM hepáticas foram o sexo masculino (OR combinada $=0,38 ; 1 C 95 \%$ : 0,20-0,72) e o fenótipo acetilador rápido/intermediário da $\mathrm{N}$-acetiltransferase 2 (OR combinada $=0,41 ; 1 C 95 \%: 0,18-0,90)$. Há, portanto, evidências para subsidiar 0 manejo de RAM antituberculose nos serviços de saúde pública.

Descritores: Tuberculose; Efeitos colaterais e reações adversas relacionados a medicamentos; Antituberculosos; Revisão.

\section{Introduction}

There have been few technological advances in the treatment of tuberculosis (TB). First-line antituberculosis drugs (rifampin, isoniazid, pyrazinamide, and ethambutol) constitute the main therapeutic strategy to control the disease, their efficacy being greater than $95 \%$ in susceptible patients. ${ }^{(1)}$

The treatment regimen currently used in developing countries is a fixed-dose, single-tablet combination of four drugs (rifampin, isoniazid, pyrazinamide, and ethambutol) in the intensive phase of treatment in order to reduce primary resistance to the isoniazid-rifampin combination and improve patient adherence to treatment. ${ }^{(1,2)}$ However, treatment discontinuation and dropout persist and result in increased morbidity and mortality from $\mathrm{TB}^{(3,4)}$

Negative outcomes of TB treatment pose risks to individual and public health, prolonging the infection and thus increasing the possibility of transmission of multidrug-resistant bacilli. Therefore, factors associated with treatment failure have been studied in order to improve treatment and prognosis. ${ }^{(5)}$

Adverse drug reactions (ADRs) are defined by the World Health Organization. ${ }^{(6)}$ The Brazilian

*Study carried out at the Federal University of Espírito Santo, Vitória, Brazil.

Correspondence to: Edson Theodoro dos Santos-Neto. Programa de Pós-Graduação em Saúde Coletiva, Centro de Ciências da Saúde, Universidade Federal do Espírito Santo. Av. Marechal Campos, 1468, Maruípe, CEP 29043-260, Vitória, ES, Brasil.

Tel. 5527 3335-7225. E-mail: edsontheodoro@uol.com.br

Financial support: None.

Submitted: 23 May 2014. Accepted, after review: 5 January 2015. 
National Ministry of Health divides adverse reactions to antituberculosis drugs into two large groups on the basis of their severity. Minor adverse effects occur in 5-20\% of cases and are thus classified because they require no immediate modification of the standard regimen and, in most cases, call for measures that can be taken at primary care clinics. Major adverse effects are less common; that is, they occur in 3-8\% of cases and call for treatment discontinuation or modification, as well as specialized care. ${ }^{(1,7)}$

According to the Brazilian guidelines on TB, adverse reactions to antituberculosis drugs are multifactorial. However, the major determinants of adverse reactions to antituberculosis drugs are the doses, the time of day at which the drugs are administered, age (from the fourth decade of life onward), nutritional status (body weight loss $>15 \%$ ), alcohol consumption (daily alcohol intake $>101 \mathrm{~mL}$ ), liver function, kidney function, and co-infection with HIV. ${ }^{(1,8,9)}$

In recent decades, there has been an increasing concern over patient adherence to antituberculosis treatment. ${ }^{(10)}$ Therefore, studies on this topic are warranted, given that adverse reactions during TB treatment are a major factor for treatment nonadherence. The objective of the present study was to identify scientific evidence on risk factors associated with adverse reactions to antituberculosis drugs.

\section{Methods}

This was a systematic review of studies retrieved from electronic databases and examining risk factors associated with adverse reactions to antituberculosis drugs. The LILACS database was searched for articles published between January of 1982 and April of 2013, and the MEDLINE database was searched for articles published between January of 1965 and April of 2013. The MEDLINE and LILACS databases were searched with the use of MeSH and DeCS terms, respectively, in combination with the appropriate Boolean operators (OR, AND, and NOT), as shown in Chart 1.

Because our database searches covered a long period, there was no need for cross-referencing. The LILACS database was concomitantly searched for articles, theses, and dissertations, articles being chosen whenever there was a duplicate entry.

The retrieved articles were initially screened by the reading of their titles and abstracts. The inclusion criteria were as follows: having an available abstract; having been published in English, Spanish, or Portuguese; being a study of humans only; being an original, quantitative study (i.e., systematic reviews, meta-analyses, and case reports being excluded); having included individuals over 10 years of age, given that the treatment regimen recommended for children differs from that recommended for adults; having included individuals with non-multidrugresistant pulmonary TB caused by Mycobacterium tuberculosis, including those with latent TB; having involved at least one of the drugs that constitute the treatment regimen recommended by the World Health Organization and the Brazilian National Ministry of Health (i.e., rifampin, isoniazid, pyrazinamide, and ethambutol); and

Chart 1 - Search strategies used in order to retrieve articles from the databases.

\begin{tabular}{|c|c|}
\hline Database & Search strategy \\
\hline MEDLINE & 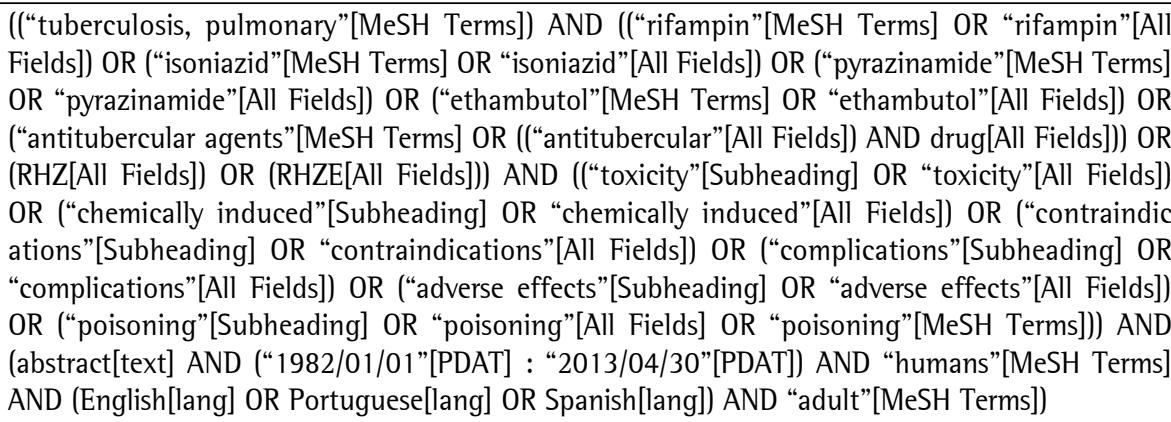 \\
\hline LILACS & $\begin{array}{l}\text { "tuberculose pulmonar" [Descritor de assunto] and rifampicina or "isoniazida" or "pirazinamida" or } \\
\text { "etambutol" or "drogas antituberculose" or "RHZ" or "RHZE" [Descritor de assunto] and "reacao } \\
\text { adversa a medicamento" or "intoxicacao" or "toxicidade" or "/efeitos adversos" or efeito colateral } \\
\text { [Palavras] }\end{array}$ \\
\hline
\end{tabular}


having described or referred to estimators of the association between ADRs and risk factors.

For each criterion, there were two response options: yes and no. In order to be included in the review, an article had to meet all of the inclusion criteria; that is, the response option for each of the aforementioned criteria had to be "yes". When there was uncertainty as to whether a given article met all of the inclusion criteria, the article in question was read in its entirety. A total of 85 articles ( 2 articles from the LILACS database and 83 articles from the MEDLINE database) were selected. Only 1 article was unavailable in full text.

In most of the studies, statistical associations between ADRs and their risk factors had not been calculated. Therefore, the data were processed with Epi Info, version 3.5.3, ORs being calculated as measures of association between risk factors and ADRs, with 95\% Cls. In addition, Pearson's chi-square test (with Yates' correction) or two-tailed Fisher's exact test was used ( $\mathrm{n}<$ 5). Meta-analyses were performed by means of the Mantel-Haenszel test and by calculating the combined OR (cOR). For all analyses, the level of significance was set at 5\%.

\section{Results}

A total of 1,389 articles were retrieved. Of those, 20 were retrieved from the LILACS database and 1,369 were retrieved from the MEDLINE database. There were 2 duplicate studies. The duplicates were excluded from the analysis, and 1,387 studies were evaluated.

Eighty-four articles were selected to be read in their entirety by two independent raters, who took into account four criteria: 1) the study did include individuals over 10 years of age, given that most of the abstracts provided no such information; 2) the study examined adverse reactions to antituberculosis drugs using the terms ADR(s), side effect(s), toxicity, or adverse effect(s); 3) the study had a loss of less than 20\% of the sample for the analyses of interest; and 4) the study included measures of association between ADRs and risk factors or allowed the calculation of such measures. In order to be included in the review, an article had to meet all four criteria.

A total of 68 articles were excluded. Of those, 21 were excluded for not meeting the inclusion criteria: much of the information that was unclear in the abstracts was clarified only after the articles were read in their entirety; 14 were excluded because they included individuals under 10 years of age; 6 were excluded for not having described any adverse reaction to antituberculosis drugs; 21 were excluded because more than 20\% of the sample was lost; and 6 were excluded for not including measures of association between ADRs and risk factors or for not including data that allowed the calculation of such measures. This resulted in the inclusion of 16 articles in the present review. The articles were selected with the aid of Microsoft Office Excel 2010, and the process is illustrated in Figure 1.

Most (63\%) of the studies were conducted in Asia, 2 were conducted in Latin America, 2 were conducted in North America, 1 was conducted in Europe, and 1 was conducted in Africa. Table 1 presents the general characteristics of the studies included in the review.

Of the 16 studies included in the present review, 56\% had been conducted in the past 10 years, and 4 had been conducted in the 1980s or 1990s. All of the studies were available in English. In addition, 63\% had a longitudinal design, and only 2 were clinical trials.

The sample sizes were measured for the proposed designs. The smallest sample consisted of 100 individuals, ${ }^{(11,12)}$ and the largest consisted of 908 individuals. ${ }^{(13)}$

Two studies involved individuals with latent TB receiving chemoprophylaxis. The treatment given to individuals with active TB varied across studies in terms of drugs, doses, frequency, and duration.

The age of the participants varied widely across studies, being unspecified in most. (14-20) Approximately 70\% of the studies involved inpatients, ${ }^{(11,14-18,20-25)}$ others involved outpatients, ${ }^{(21,26)}$ and 1 involved an unspecified population. ${ }^{(19)}$

Statistical analysis revealed that the risk factors associated with adverse reactions to antituberculosis drugs were as follows: gender; race/ethnicity; nationality; age; weight; marital status; treatment regimen; genetic factors; anemia; co-infection with HIV, HBV, or HCV; diabetes; liver disease; hypoalbuminemia; hyponatremia; lymphopenia; and alcohol, tobacco, or illicit drug use.

Table 2 presents the risk factors that were significantly associated with ADRs. The ADRs were 


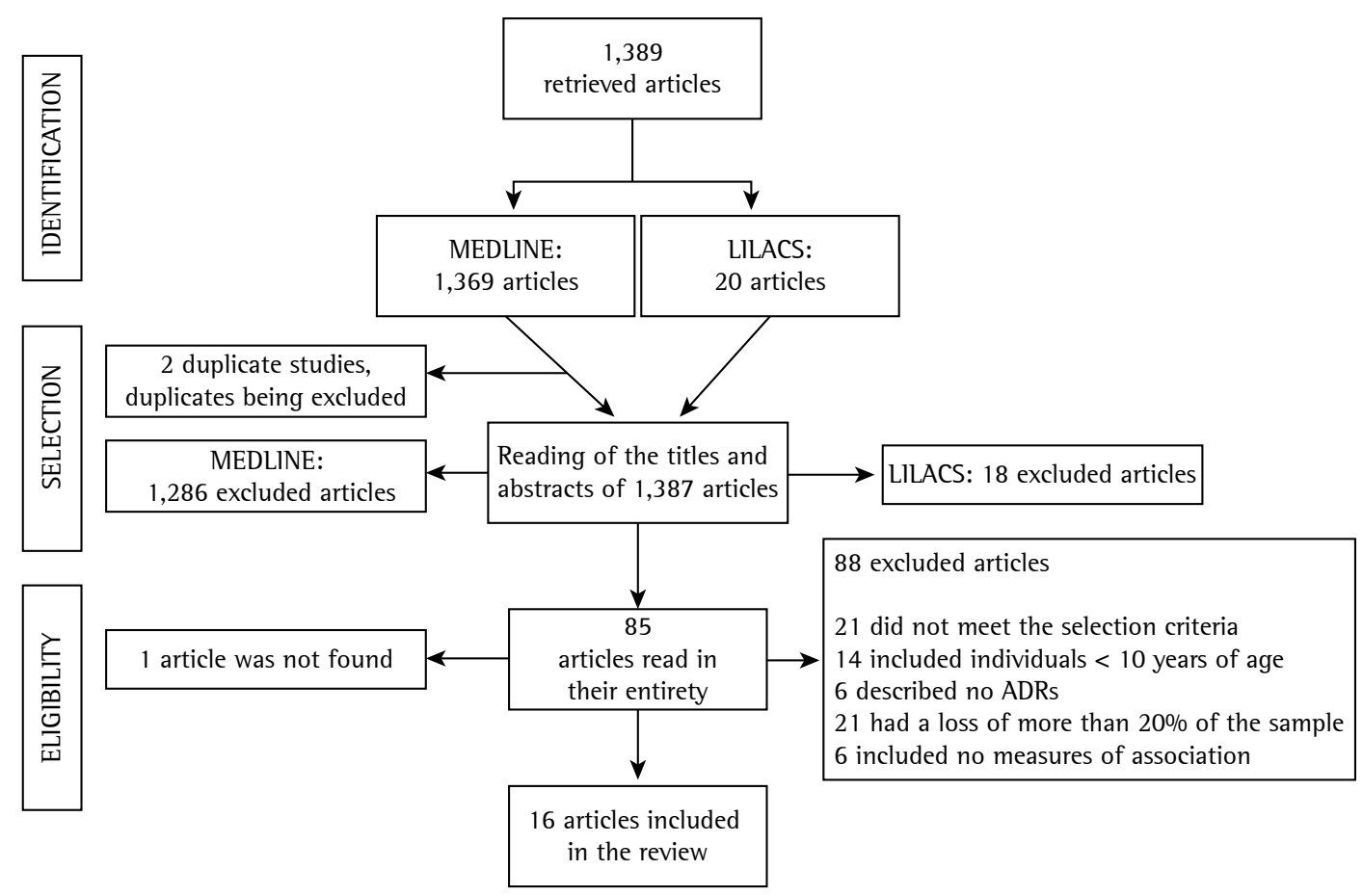

Figure 1 - Flowchart of article selection. ADRs: adverse drug reactions.

divided into gastrointestinal ADRs, neurological ADRs, immune-mediated ADRs, hepatic ADRs, and other ADRs.

Gastrointestinal ADRs included nausea, vomiting, indigestion, diarrhea, and other, unspecified, reactions. Co-infection with HIV was the only risk factor for gastrointestinal ADRs, with a significant association with the development of diarrhea. ${ }^{(20)}$

Neurological ADRs included vertigo and other, unspecified, reactions. However, no risk factors were significantly associated with neurological ADRs.

Immune-mediated ADRs included fever, herpes zoster, Kaposi's sarcoma, oral candidiasis, immune reconstitution inflammatory syndrome, and other, unspecified, reactions. Co-infection with HIV was found to be a risk factor for Kaposi's sarcoma and oral candidiasis. ${ }^{(20)}$ Fever was a common ADR, being significantly associated with the following risk factors: anemia; hypoalbuminemia; hyponatremia; alcoholism; and co-infection with HIV. ${ }^{(16)}$

Hepatic ADRs were the most investigated ADRs, including jaundice, hepatitis, hepatotoxicity, and drug-induced hepatotoxicity. The rifampinstreptomycin-isoniazid-pyrazinamide combination taken daily for 3 months; the rifampin- streptomycin-isoniazid-pyrazinamide taken daily for 3 months, followed by the streptomycinisoniazid-pyrazinamide combination taken twice a week for 2 months; and the streptomycinisoniazid-pyrazinamide combination taken daily for 3 months, followed by the streptomycinisoniazid-pyrazinamide combination taken twice a week for 2 months were compared and were found to be statistically significant protective factors for jaundice. ${ }^{(13)}$ In addition, primary chemoprophylaxis was found to be a statistically significant protective factor for hepatotoxicity. ${ }^{(21)}$

The association between genetic factors and hepatic ADRs was evaluated in 5 studies. A slow $\mathrm{N}$-acetyltransferase 2 (NAT2) acetylator phenotype was found to be a significant risk factor for hepatitis. ${ }^{(25)}$ In contrast, a rapid/intermediate NAT2 acetylator phenotype was found to be a protective factor for hepatotoxicity. ${ }^{(11,18)} \mathrm{In}$ addition, a C/C genotype at rs2070401 in BACH1 and a G/A or A/A genotype at rs4720833 in MAFK were found to be risk factors for drug-induced hepatotoxicity. ${ }^{(12)}$

Only 3 studies showed a statistically significant association between sociodemographic factors and hepatic ADRs. One study showed that being under 65 years of age is a protective factor for hepatitis, ${ }^{(15)}$ whereas another showed that 
Table 1 - Studies included in the systematic review, conducted in the 1965-2012 period.

\begin{tabular}{|c|c|c|c|c|c|c|c|c|}
\hline Author & Study design & $\begin{array}{l}\text { Age } \\
\text { group } \\
\text { (years) }\end{array}$ & $\begin{array}{l}\text { Sample } \\
\text { (n) }\end{array}$ & $\begin{array}{c}\text { Study } \\
\text { population }\end{array}$ & Study period & $\begin{array}{l}\text { Study } \\
\text { setting }\end{array}$ & $\begin{array}{c}\text { Treatment } \\
\text { regimen }\end{array}$ & $\begin{array}{l}\text { Statistical } \\
\text { analysis }\end{array}$ \\
\hline $\begin{array}{l}\mathrm{Ai} \\
\text { et al. }{ }^{(14)}\end{array}$ & $\begin{array}{l}\text { Case-control } \\
\text { study }\end{array}$ & $\begin{array}{c}\leq 60 \text { and } \\
>60\end{array}$ & 639 & Outpatients & $\begin{array}{c}\text { June of } 2006 \\
\text { to March of } \\
2007\end{array}$ & China & $\begin{array}{l}\text { RHZE for } \\
2 \text { months } \\
+ \text { RH for } \\
4 \text { months }\end{array}$ & $\begin{array}{l}\text { Unspecified } \\
\text { univariate } \\
\text { and } \\
\text { multivariate } \\
\text { analyses }\end{array}$ \\
\hline $\begin{array}{l}\text { Baghaei } \\
\text { et al. }{ }^{(15)}\end{array}$ & $\begin{array}{l}\text { Population- } \\
\text { based cohort } \\
\text { study }\end{array}$ & $\begin{array}{c}<65 \text { and } \\
\geq 65\end{array}$ & 761 & Inpatients & $\begin{array}{c}\text { January of } \\
2006 \text { to } \\
\text { January of } \\
2008\end{array}$ & Iran & $\begin{array}{l}\text { RHZE for } \\
6 \text { months }\end{array}$ & $\begin{array}{l}\mathrm{X}^{2} \text {, Fisher's } \\
\text { exact test, } \\
\text { Mann- } \\
\text { Whitney } \\
\text { test, and } \\
\text { logistic } \\
\text { regression }\end{array}$ \\
\hline $\begin{array}{l}\text { Barnes } \\
\text { et al. }{ }^{(16)}\end{array}$ & Cohort study & $\geq 15$ & 161 & Inpatients & $\begin{array}{c}\text { June of } 1984 \\
\text { to March of } \\
1985\end{array}$ & USA & No data & $\begin{array}{l}\mathrm{X}^{2} \text { and } \\
\text { Fisher's } \\
\text { exact test }\end{array}$ \\
\hline $\begin{array}{l}\text { Cantalice } \\
\text { Filho } \\
\text { et al. } .^{(17)}\end{array}$ & $\begin{array}{l}\text { Case-control } \\
\text { study }\end{array}$ & $\begin{array}{c}15-49 \\
\text { and } \geq 60\end{array}$ & 581 & Inpatients & $\begin{array}{l}\text { January of } \\
1980 \text { to } \\
\text { December of } \\
1996\end{array}$ & Brazil & $\begin{array}{c}\text { RHZ for } 2 \\
\text { months }+ \\
\text { RH for } 4 \\
\text { months }\end{array}$ & $\begin{array}{l}\mathrm{X}^{2} \text { and } \\
\text { Fisher's } \\
\text { exact test }\end{array}$ \\
\hline $\begin{array}{l}\text { Cho } \\
\text { et al. }{ }^{(18)}\end{array}$ & Cohort study & $\begin{array}{c}51.2 \pm \\
17.5 \\
46.7 \pm \\
18.4\end{array}$ & 132 & Inpatients & $\begin{array}{l}\text { June of } 2004 \\
\text { to December } \\
\text { of } 2005\end{array}$ & $\begin{array}{l}\text { South } \\
\text { Korea }\end{array}$ & $\begin{array}{l}\text { RHZE for } \\
2 \text { months } \\
+ \text { HRE for } \\
4 \text { months }\end{array}$ & $\begin{array}{c}\text { Mann- } \\
\text { Whitney } \\
\text { test, } X^{2}, \\
\text { and Fisher's } \\
\text { exact test }\end{array}$ \\
\hline $\begin{array}{l}\text { HKCS/ } \\
\text { BMRC }^{(19)}\end{array}$ & Clinical trial & $\geq 15$ & 620 & Unspecified & $\begin{array}{c}\text { October of } \\
1984 \text { to } \\
\text { October of } \\
1986\end{array}$ & China & $\begin{array}{c}\text { SHRZ }(3 \times 1 \\
\text { week for } 6 \\
\text { months) }\end{array}$ & $\begin{array}{c}\text { Relative } \\
\text { frequency } \\
(\%) \text { and } \\
\text { absolute } \\
\text { frequency } \\
\text { (n), } \\
\text { unspecified } \\
\text { univariate } \\
\text { analysis }\end{array}$ \\
\hline $\begin{array}{l}\text { Kelly } \\
\text { et al. }{ }^{(20)}\end{array}$ & Cohort study & $\begin{array}{c}\text { Mean, } \\
34.9 \text { and } \\
41.7\end{array}$ & 187 & Inpatients & $\begin{array}{c}\text { November of } \\
1991 \text { to May } \\
\text { of } 1993\end{array}$ & Africa & $\begin{array}{l}\text { SHRZ for } \\
2 \text { months/ } \\
\text { TH for } 6 \\
\text { months }\end{array}$ & $\begin{array}{l}\text { Kaplan- } \\
\text { Meier } \\
\text { method, } \\
\text { unspecified } \\
\text { univariate } \\
\text { and } \\
\text { multivariate } \\
\text { analyses }\end{array}$ \\
\hline $\begin{array}{l}\text { Khalili } \\
\text { et al. }{ }^{(11)}\end{array}$ & $\begin{array}{l}\text { Case-control } \\
\text { study }\end{array}$ & $18-86$ & 100 & Inpatients & $\begin{array}{c}\text { September } \\
\text { of } 2006 \text { to } \\
\text { March of } \\
2009\end{array}$ & Iran & $\begin{array}{l}\text { RHZE for } \\
2 \text { months } \\
+ \text { RH for } \\
4 \text { months }\end{array}$ & $\mathrm{X}^{2}$ \\
\hline $\begin{array}{l}\text { Lee } \\
\text { et al. }{ }^{(21)}\end{array}$ & $\begin{array}{l}\text { Retrospective } \\
\text { cohort study }\end{array}$ & $18-84$ & $\begin{array}{c}148 \\
\text { (Latent } \\
\text { TB) }\end{array}$ & $\begin{array}{l}\text { Outpatients/ } \\
\text { inpatients }\end{array}$ & $\begin{array}{l}\text { April of } 1999 \\
\text { to March of } \\
2001\end{array}$ & USA & $\begin{array}{l}\text { RZ for } 2 \\
\text { months }\end{array}$ & $\begin{array}{l}\text { Relative } \\
\text { frequency } \\
(\%) \text { and } \\
\text { absolute } \\
\text { frequency } \\
\text { (n), relative } \\
\text { risk, and } \\
\text { multivariate } \\
\text { analysis }\end{array}$ \\
\hline
\end{tabular}


Table 1 - Continued...

\begin{tabular}{|c|c|c|c|c|c|c|c|c|}
\hline Author & Study design & $\begin{array}{l}\text { Age } \\
\text { group } \\
\text { (years) }\end{array}$ & $\begin{array}{l}\text { Sample } \\
\text { (n) }\end{array}$ & $\begin{array}{c}\text { Study } \\
\text { population }\end{array}$ & Study period & $\begin{array}{l}\text { Study } \\
\text { setting }\end{array}$ & $\begin{array}{l}\text { Treatment } \\
\text { regimen }\end{array}$ & $\begin{array}{l}\text { Statistical } \\
\text { analysis }\end{array}$ \\
\hline $\begin{array}{l}\text { Nanashima } \\
\text { et al.(12) }\end{array}$ & $\begin{array}{l}\text { Randomized } \\
\text { cross- } \\
\text { sectional } \\
\text { study }\end{array}$ & $22-94$ & 100 & Inpatients & $\begin{array}{l}\text { May of } 2005 \\
\text { to September } \\
\text { of } 2006\end{array}$ & Japan & $\begin{array}{l}\mathrm{H} \text { (400 } \\
\text { mg/day) } \\
+\mathrm{R}(450 \\
\text { mg/day) }\end{array}$ & $\begin{array}{l}\text { Mann- } \\
\text { Whitney } \\
\text { test, } X^{2} \text {, } \\
\text { Fisher's } \\
\text { exact test, } \\
\text { and logistic } \\
\text { regression }\end{array}$ \\
\hline $\begin{array}{l}\text { Martínez } \\
\text { Sanchís } \\
\text { et al. }{ }^{(22)}\end{array}$ & Cohort study & $\begin{array}{l}\geq 10 \text { to } \\
\geq 64\end{array}$ & $\begin{array}{l}198 \\
\text { (Latent } \\
\text { TB) }\end{array}$ & Inpatients & $\begin{array}{l}\text { December } \\
\text { of } 1996 \text { to } \\
\text { December of } \\
2002\end{array}$ & Spain & $\begin{array}{l}\mathrm{H} \\
300 \mathrm{mg} / \\
\text { day (2 or } \\
6 \text { months) }\end{array}$ & $\begin{array}{l}\mathrm{X}^{2} \text {, Fisher's } \\
\text { exact test, } \\
\text { and logistic } \\
\text { regression }\end{array}$ \\
\hline $\begin{array}{l}\text { Sharma } \\
\text { et al. }{ }^{(23)}\end{array}$ & Cohort study & $16-80$ & 346 & Inpatients & 1996-2000 & India & RHZE & $\begin{array}{l}\mathrm{X}^{2} \text { and } \\
\text { logistic } \\
\text { regression }\end{array}$ \\
\hline $\begin{array}{l}\text { Sirinak } \\
\text { et al. } .^{(24)}\end{array}$ & Cohort study & $\geq 18$ & 769 & Inpatients & $\begin{array}{l}\text { May of } 2005 \\
\text { to September } \\
\text { of } 2006\end{array}$ & Thailand & RHZE & $\begin{array}{l}\text { Unspecified } \\
\text { univariate } \\
\text { analysis } \\
\text { and logistic } \\
\text { regression }\end{array}$ \\
\hline $\begin{array}{l}\text { Teixeira } \\
\text { et al. }{ }^{(25)}\end{array}$ & $\begin{array}{l}\text { Case-control } \\
\text { study }\end{array}$ & $>18$ & 167 & Inpatients & 1998-2008 & Brazil & $\begin{array}{l}\mathrm{H}(400 \\
\mathrm{mg} / \text { day })+ \\
\text { others }\end{array}$ & $\begin{array}{l}\text { Mann- } \\
\text { Whitney } \\
\text { test, X' } \\
\text { Fisher's } \\
\text { exact test, } \\
\text { Student's } \\
\text { t-test, etc. }\end{array}$ \\
\hline $\begin{array}{l}\text { Teleman } \\
\text { et al. }{ }^{(26)}\end{array}$ & $\begin{array}{l}\text { Retrospective } \\
\text { cohort study }\end{array}$ & $16-82$ & 783 & Outpatients & $\begin{array}{l}\text { January of } \\
1998 \text { to } \\
\text { December of } \\
1998\end{array}$ & Singapore & $\begin{array}{l}\text { RHZ for } 9 \\
\text { months }+ \\
\text { E or } S\end{array}$ & $\begin{array}{l}\text { Mann- } \\
\text { Whitney } \\
\text { test, X', } \\
\text { Fisher's } \\
\text { exact test, } \\
\text { and logistic } \\
\text { regression }\end{array}$ \\
\hline $\begin{array}{l}\text { No authors } \\
\text { listed }^{(13)}\end{array}$ & Clinical trial & $\geq 12$ & 908 & Outpatients & Unspecified & India & R3/R5/Z5 & $\begin{array}{l}\text { Relative } \\
\text { frequency } \\
(\%) \text { and } \\
\text { absolute } \\
\text { frequency } \\
\text { (n), } \\
\text { unspecified } \\
\text { univariate } \\
\text { analysis }\end{array}$ \\
\hline
\end{tabular}

TB: tuberculosis; HKCS: Hong Kong Chest Service; BMRC: British Medical Research Council; R: rifampin; H: isoniazid; Z: pyrazinamide; E: ethambutol; S: streptomycin; T: thiocetazone; R3: rifampin-streptomycin-isoniazid-pyrazinamide daily for 3 months; R5: rifampin-streptomycin-isoniazid-pyrazinamide daily for 3 months, followed by streptomycinisoniazid-pyrazinamide twice a week for 2 months; Z5: streptomycin-isoniazid-pyrazinamide daily for 3 months, followed by streptomycin-isoniazid-pyrazinamide twice a week for 2 months; and $\mathrm{X}^{2}$ : chi-square test.

being $\leq 60$ years of age is a protective factor for drug-induced hepatotoxicity. ${ }^{(26)}$ Being male was found to be a significant protective factor for hepatotoxicity. ${ }^{(21)}$

Other ADRs included arthralgia, exanthema, and unspecified reactions (kidney disorders, jaundice, hearing loss, liver problems, and skin rash). One study showed that age $(<60$ years $)$ was a significant protective factor for unspecified reactions. ${ }^{(14)}$ The rifampin-streptomycin-isoniazidpyrazinamide combination taken daily for 3 months and the rifampin-streptomycin-isoniazidpyrazinamide taken daily for 3 months, followed by the streptomycin-isoniazid-pyrazinamide combination taken twice a week for 2 months were protective factors for arthralgia; in contrast, 
Table 2 - Statistically significant associations between risk factors and adverse effects of antituberculosis drugs in the reviewed studies, conducted in the 1965-2013 period.

\begin{tabular}{|c|c|c|c|c|c|c|c|}
\hline Author & Risk factor & $A$ & $\overline{D R}$ & OR & $95 \% \mathrm{Cl}$ & $\begin{array}{c}\mathrm{X}^{2} \text { (Yates' } \\
\text { correction) } \\
\text { or two-tailed } \\
\text { Fisher's exact } \\
\text { test } \\
\end{array}$ & $\begin{array}{l}\text { Value } \\
\text { of } p\end{array}$ \\
\hline $\begin{array}{l}\text { Kelly } \\
\text { et al. }{ }^{(20)}\end{array}$ & $\begin{array}{l}\text { HIV } \\
\text { Present } \\
\text { Absent }\end{array}$ & $\begin{array}{c}\text { Diarrhea }+ \\
42 \\
10\end{array}$ & $\begin{array}{c}\text { Diarrhea - } \\
83 \\
52\end{array}$ & 2.63 & $1.17-6.37$ & 5.461 & 0.019 \\
\hline $\begin{array}{l}\text { Barnes } \\
\text { et al. }{ }^{(16)}\end{array}$ & $\begin{array}{l}\text { Hyponatremia } \\
\text { Present } \\
\text { Absent }\end{array}$ & $\begin{array}{c}\text { Fever }+ \\
9 \\
5\end{array}$ & $\begin{array}{c}\text { Fever - } \\
22 \\
51\end{array}$ & 16.6 & $3.95-146.80$ & $\begin{array}{l}\text { Two-tailed } \\
\text { Fisher's exact } \\
\text { test }\end{array}$ & 0.000 \\
\hline $\begin{array}{l}\text { Barnes } \\
\text { et al. }{ }^{(16)}\end{array}$ & $\begin{array}{l}\text { Hypoalbuminemia } \\
\text { Present } \\
\text { Absent }\end{array}$ & $\begin{array}{c}\text { Fever }+ \\
88 \\
18\end{array}$ & $\begin{array}{c}\text { Fever - } \\
24 \\
30\end{array}$ & 6.11 & $2.74-13.68$ & 23.545 & 0.000 \\
\hline $\begin{array}{l}\text { Barnes } \\
\text { et al. }{ }^{(16)}\end{array}$ & $\begin{array}{l}\text { Alcoholism } \\
\text { Present } \\
\text { Absent }\end{array}$ & $\begin{array}{c}\text { Fever }<7 \text { days } \\
10 \\
4\end{array}$ & $\begin{array}{c}\text { Fever }>7 \text { days } \\
23 \\
48\end{array}$ & 5.22 & $1.30-24.76$ & $\begin{array}{l}\text { Two-tailed } \\
\text { Fisher's exact } \\
\text { test }\end{array}$ & 0.014 \\
\hline $\begin{array}{l}\text { Barnes } \\
\text { et al. }{ }^{(16)}\end{array}$ & $\begin{array}{l}\text { Anemia } \\
\text { Present } \\
\text { Absent }\end{array}$ & $\begin{array}{c}\text { Fever }<7 \text { days } \\
14 \\
0\end{array}$ & $\begin{array}{c}\text { Fever }>7 \text { days } \\
54 \\
59\end{array}$ & $\infty$ & 3.3804 to $\infty$ & $\begin{array}{l}\text { Two-tailed } \\
\text { Fisher's exact } \\
\text { test }\end{array}$ & 0.000 \\
\hline $\begin{array}{l}\text { Barnes } \\
\text { et al. }{ }^{(16)}\end{array}$ & $\begin{array}{l}\text { Hyponatremia } \\
\text { Present } \\
\text { Absent }\end{array}$ & $\begin{array}{c}\text { Fever }<7 \text { days } \\
9 \\
5\end{array}$ & $\begin{array}{c}\text { Fever }>7 \text { days } \\
22 \\
51\end{array}$ & 4.17 & $1.09-17.46$ & 4.577 & 0.032 \\
\hline $\begin{array}{l}\text { Barnes } \\
\text { et al. }{ }^{(16)}\end{array}$ & $\begin{array}{l}\text { Hypoalbuminemia } \\
\text { Present } \\
\text { Absent }\end{array}$ & $\begin{array}{c}\text { Fever }<7 \text { days } \\
14 \\
0\end{array}$ & $\begin{array}{c}\text { Fever }>7 \text { days } \\
54 \\
19\end{array}$ & $\infty$ & 1.03 to $\infty$ & $\begin{array}{l}\text { Two-tailed } \\
\text { Fisher's exact } \\
\text { test }\end{array}$ & 0.034 \\
\hline $\begin{array}{l}\text { Kelly } \\
\text { et al. }{ }^{(20)}\end{array}$ & $\begin{array}{l}\text { HIV } \\
\text { Present } \\
\text { Absent }\end{array}$ & $\begin{array}{c}\text { Fever }+ \\
69 \\
20\end{array}$ & $\begin{array}{c}\text { Fever - } \\
56 \\
42\end{array}$ & 2.59 & $1.31-5.19$ & 7.850 & 0.005 \\
\hline $\begin{array}{l}\text { Kelly } \\
\text { et al. }{ }^{(20)}\end{array}$ & $\begin{array}{l}\text { HIV } \\
\text { Present } \\
\text { Absent }\end{array}$ & $\begin{array}{c}\text { Oral candidiasis + } \\
19 \\
1\end{array}$ & $\begin{array}{c}\text { Oral candidiasis - } \\
106 \\
61\end{array}$ & 10.93 & $1.64-461.62$ & $\begin{array}{l}\text { Two-tailed } \\
\text { Fisher's exact } \\
\text { test }\end{array}$ & 0.004 \\
\hline $\begin{array}{l}\text { Kelly } \\
\text { et al. }{ }^{(20)}\end{array}$ & $\begin{array}{l}\text { HIV } \\
\text { Present } \\
\text { Absent }\end{array}$ & $\begin{array}{c}\text { Kaposi's sarcoma }+ \\
10 \\
0\end{array}$ & $\begin{array}{c}\text { Kaposi's sarcoma - } \\
115 \\
62\end{array}$ & $\infty$ & 1.15 to $\infty$ & $\begin{array}{l}\text { Two-tailed } \\
\text { Fisher's exact } \\
\text { test }\end{array}$ & 0.032 \\
\hline Ai et al. ${ }^{(14)}$ & $\begin{array}{l}\text { Age } \\
<60 \text { years } \\
>60 \text { years }\end{array}$ & $\begin{array}{c}\mathrm{ADR}+ \\
209 \\
77\end{array}$ & $\begin{array}{c}\mathrm{ADR}- \\
288 \\
65\end{array}$ & 0.61 & $0.41-0.91$ & 6.136 & 0.013 \\
\hline $\begin{array}{l}\text { Lee } \\
\text { et al. }{ }^{(21)}\end{array}$ & $\begin{array}{l}\text { Recent infection } \\
\text { Present } \\
\text { Absent }\end{array}$ & $\begin{array}{c}\text { Hepatotoxicity + } \\
13 \\
1\end{array}$ & $\begin{array}{c}\text { Hepatotoxicity - } \\
66 \\
68\end{array}$ & 13.39 & $1.89-577.38$ & $\begin{array}{l}\text { Two-tailed } \\
\text { Fisher's exact } \\
\text { test }\end{array}$ & 0.002 \\
\hline $\begin{array}{l}\text { No authors } \\
\text { listed }^{(13)}\end{array}$ & $\begin{array}{l}\text { Therapeutic regimen } \\
\text { R3 } \\
\text { R5 and Z5 }\end{array}$ & $\begin{array}{c}\text { Jaundice }+ \\
18 \\
328\end{array}$ & $\begin{array}{c}\text { Jaundice - } \\
279 \\
283\end{array}$ & 0.06 & $0.03-0.09$ & 190.15 & 0.000 \\
\hline $\begin{array}{l}\text { No authors } \\
\text { listed }^{(13)}\end{array}$ & $\begin{array}{l}\text { Therapeutic } \\
\text { regimen } \\
\text { R5 } \\
\text { R3 and Z5 }\end{array}$ & $\begin{array}{c}\text { Jaundice + } \\
26 \\
320\end{array}$ & $\begin{array}{c}\text { Jaundice - } \\
281 \\
281\end{array}$ & 0.08 & $0.05-0.13$ & 170.83 & 0.000 \\
\hline $\begin{array}{l}\text { No authors } \\
\text { listed }^{(13)}\end{array}$ & $\begin{array}{l}\text { Therapeutic } \\
\text { regimen } \\
\text { Z5 } \\
\text { R3 and R5 }\end{array}$ & $\begin{array}{c}\text { Jaundice }+ \\
2 \\
344\end{array}$ & $\begin{array}{c}\text { Jaundice - } \\
302 \\
260\end{array}$ & 0.01 & $0.00-0.02$ & $\begin{array}{l}\text { Two-tailed } \\
\text { Fisher's exact } \\
\text { test }\end{array}$ & 0.000 \\
\hline $\begin{array}{l}\text { Cho } \\
\text { et al. }{ }^{(18)}\end{array}$ & $\begin{array}{l}\text { NAT2 } \\
\text { Rapid or } \\
\text { intermediate } \\
\text { acetylator } \\
\text { Slow acetylator }\end{array}$ & $\begin{array}{c}\text { Hepatotoxicity + } \\
11 \\
7 \\
\end{array}$ & $\begin{array}{c}\text { Hepatotoxicity - } \\
102 \\
12 \\
\end{array}$ & 0.18 & $0.05-0.68$ & 7.977 & 0.005 \\
\hline
\end{tabular}


Table 2 - Continued...

\begin{tabular}{|c|c|c|c|c|c|c|c|}
\hline Author & Risk factor & $\mathrm{AI}$ & DR & OR & $95 \% \mathrm{Cl}$ & $\begin{array}{c}\mathrm{X}^{2} \text { (Yates' } \\
\text { correction) } \\
\text { or two-tailed } \\
\text { Fisher's exact } \\
\text { test }\end{array}$ & $\begin{array}{l}\text { Value } \\
\text { of } p\end{array}$ \\
\hline $\begin{array}{l}\text { Khalili } \\
\text { et al. }{ }^{(11)}\end{array}$ & $\begin{array}{l}\text { NAT2 } \\
\text { Rapid or } \\
\text { intermediate } \\
\text { acetylator } \\
\text { Slow acetylator }\end{array}$ & $\begin{array}{l}\text { Hepatotoxicity }+ \\
\qquad \begin{array}{c}5 \\
9\end{array}\end{array}$ & $\begin{array}{l}\text { Hepatotoxicity - } \\
\qquad \begin{array}{c}31 \\
5\end{array}\end{array}$ & 0.09 & $0.02-0.46$ & 10.322 & 0.001 \\
\hline $\begin{array}{l}\text { Nanashima } \\
\text { et al. }{ }^{(12)}\end{array}$ & $\begin{array}{l}\text { C/C genotype } \\
\text { at rs2070401 in } \\
\text { BACH1 } \\
\text { G/A or A/A } \\
\text { genotype at } \\
\text { rs4720833 in } \\
\text { MAFK } \\
\text { Present } \\
\text { Absent }\end{array}$ & $\begin{array}{c}\text { Drug-induced } \\
\text { hepatotoxicity + }\end{array}$ & $\begin{array}{c}\text { Drug-induced } \\
\text { hepatotoxicity - }\end{array}$ & 9.73 & $2.04-90.86$ & $\begin{array}{l}\text { Two-tailed } \\
\text { Fisher's exact } \\
\text { test }\end{array}$ & 0.001 \\
\hline $\begin{array}{l}\text { Teixeira } \\
\text { et al. }{ }^{(25)}\end{array}$ & $\begin{array}{l}\text { NAT2 genotype } \\
\text { Slow acetylator } \\
\text { Others }\end{array}$ & $\begin{array}{c}\text { Hepatitis }+ \\
18 \\
8\end{array}$ & $\begin{array}{c}\text { Hepatitis - } \\
64 \\
77\end{array}$ & 2.71 & $1.03-7.65$ & 4.084 & 0.043 \\
\hline $\begin{array}{l}\text { Baghaei } \\
\text { et al. }{ }^{(15)}\end{array}$ & $\begin{array}{l}\text { Age } \\
<65 \text { years } \\
\geq 65 \text { years }\end{array}$ & $\begin{array}{l}\text { Hepatitis + } \\
50 \\
49\end{array}$ & $\begin{array}{c}\text { Hepatitis - } \\
416 \\
246\end{array}$ & 0.6 & $0.39-0.94$ & 5.013 & 0.025 \\
\hline $\begin{array}{l}\text { Teleman et } \\
\text { al. }{ }^{(26)}\end{array}$ & $\begin{array}{l}\text { Age } \\
\leq 60 \text { years } \\
>60 \text { years }\end{array}$ & $\begin{array}{c}\text { Drug-induced } \\
\text { hepatotoxicity }+ \\
26 \\
29\end{array}$ & $\begin{array}{c}\text { Drug-induced } \\
\text { hepatotoxicity - } \\
613 \\
368\end{array}$ & 0.04 & $0.00-0.17$ & 37.264 & 0.000 \\
\hline $\begin{array}{l}\text { No authors } \\
\text { listed }^{(13)}\end{array}$ & $\begin{array}{l}\text { Therapeutic } \\
\text { regimen } \\
\text { R3 } \\
\text { R5 and Z5 }\end{array}$ & $\begin{array}{c}\text { Arthralgia + } \\
137 \\
345\end{array}$ & $\begin{array}{c}\text { Arthralgia - } \\
160 \\
266\end{array}$ & 0.66 & $0.49-0.88$ & 8.164 & 0.004 \\
\hline $\begin{array}{l}\text { No authors } \\
\text { listed }^{(13)}\end{array}$ & $\begin{array}{l}\text { Therapeutic } \\
\text { regimen } \\
\text { R5 } \\
\text { R3 and Z5 }\end{array}$ & $\begin{array}{c}\text { Arthralgia + } \\
133 \\
349\end{array}$ & $\begin{array}{c}\text { Arthralgia - } \\
174 \\
252\end{array}$ & 0.55 & $0.41-0.74$ & 17.158 & 0.000 \\
\hline $\begin{array}{l}\text { No authors } \\
\text { listed }^{(13)}\end{array}$ & $\begin{array}{l}\text { Therapeutic } \\
\text { regimen } \\
\mathrm{Z5} \\
\mathrm{R} 3 \text { and R5 }\end{array}$ & $\begin{array}{c}\text { Arthralgia + } \\
212 \\
270 \\
\end{array}$ & $\begin{array}{c}\text { Arthralgia - } \\
92 \\
334 \\
\end{array}$ & 2.85 & $2.11-3.87$ & 49.89 & 0.000 \\
\hline
\end{tabular}

ADR: adverse drug reaction; $\mathrm{X}^{2}$ : chi-square test; R3: rifampin-streptomycin-isoniazid-pyrazinamide daily for 3 months; R5: rifampin-streptomycin-isoniazid-pyrazinamide daily for 3 months, followed by streptomycin-isoniazid-pyrazinamide twice a week for 2 months; Z5: streptomycin-isoniazid-pyrazinamide daily for 3 months, followed by streptomycinisoniazid-pyrazinamide twice a week for 2 months; and NAT2: N-acetyltransferase 2.

the streptomycin-isoniazid-pyrazinamide combination taken daily for 3 months, followed by the streptomycin-isoniazid-pyrazinamide combination taken twice a week for 2 months was a risk factor for arthralgia. ${ }^{(13)}$

The results of our meta-analyses (Figure 2) show that protective factors for hepatic ADRs include showing a rapid/intermediate NAT2 acetylator phenotype $(\mathrm{cOR}=0.41 ; 95 \% \mathrm{Cl}$ : $0.18-0.90$ ), being 35 years of age or older (cOR $=0.38 ; 95 \% \mathrm{Cl}: 0.20-0.72$ ), and being male ( $\mathrm{COR}=0.38 ; 95 \% \mathrm{Cl}: 0.20-0.72$ ).

\section{Discussion}

In the present review, one study ${ }^{(13)}$ demonstrated that a therapeutic regimen without rifampin was a risk factor for arthralgia. This finding suggests that rifampin can indirectly offer protection against arthralgia.

Joint pain is considered to be a minor side effect, and, when unrelated to hyperuricemia, it is frequently associated with pyrazinamide and, more rarely, isoniazid. ${ }^{(8)}$ This is probably due to pyrazinoic acid, which is the major metabolite 


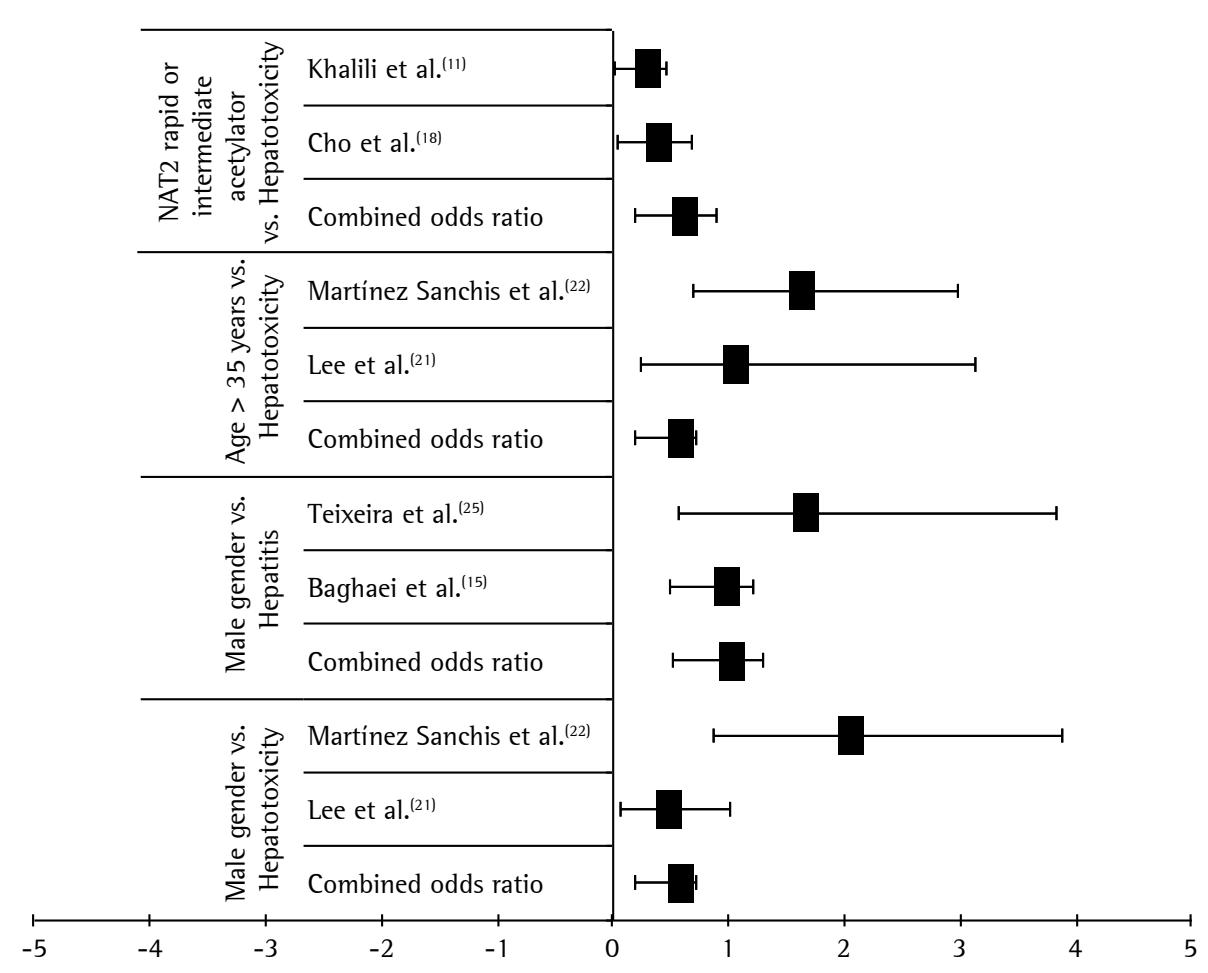

Figure 2 - Meta-analysis of the factors associated with hepatic adverse reactions to antituberculosis drugs.

of pyrazinamide; pyrazinoic acid inhibits renal tubular secretion of uric acid, thus increasing its serum concentration and causing joint pain. ${ }^{(7)}$ In a multicenter study, ${ }^{(27)}$ arthralgia was reported in 6 of 617 patients receiving rifampin, isoniazid, and pyrazinamide but in none of the 445 patients who received rifampin and isoniazid. However, most $(87.5 \%)$ of the studies included in the present review involved different treatment regimens. Therefore, the results obtained by combining these studies can be misleading, and this is a limitation of our review.

The present review included 2 studies addressing latent TB rather than active TB. One of the studies ${ }^{(22)}$ showed that primary chemoprophylaxis (the treatment of predisposed individuals in order to prevent TB infection, i.e., before they present with positive tuberculin skin test results) with $300 \mathrm{mg}$ of isoniazid and 50 $\mathrm{mg}$ of pyridoxine for 2 months was a significant protective factor for hepatotoxicity when compared with secondary chemoprophylaxis (the treatment of latent TB infection, i.e., the treatment of patients with positive tuberculin skin test results without disease), which lasted longer (i.e., 6 months).

The duration of drug exposure can be a determinant of hepatic ADRs, given that longer exposure to toxic metabolites translates to a greater chance of severe injury. A study conducted in 2000 in Barcelona, Spain, showed that the duration of chemoprophylaxis was associated with toxic effects; however, it was impossible to establish a relationship with the type of drug used. ${ }^{(28)}$

There is divergence across studies regarding the association between HIV co-infection and ADRs during TB treatment. ${ }^{(10)}$ In patients with TB/HIV co-infection, ADRs are generally related to the immune system itself ${ }^{(29)}$ and are due to immunosuppression and drug metabolism pathways, which often generate toxic compounds. Therefore, the findings of one study, ${ }^{(20)}$ in which HIV co-infection was considered a risk factor for diarrhea, are justifiable.

The interaction among antituberculosis drugs can potentiate their toxic effects on the gastrointestinal and hepatic systems. ${ }^{(22)}$ Breen et al. ${ }^{(30)}$ found no difference between groups of patients with and without HIV co-infection in terms of the incidence of hepatotoxicity. In another study, ${ }^{(29)}$ co-infection with HIV was found to be a risk factor for grade 1 hepatotoxicity, defined as a three-fold increase in the lower limit of normal for alanine aminotransferase. 
With regard to the use of alcohol, much of the alcohol ingested by humans is metabolized in the liver by the enzyme alcohol dehydrogenase. This enzyme converts alcohol to acetaldehyde, which has toxic effects even at reduced concentrations. ${ }^{(31,32)}$ There is also evidence that the induction of cytochrome P450 2E1 by ethanol is related to the pathogenesis of alcoholic liver disease. ${ }^{(33)} \mathrm{Coca}^{(29)}$ reported that alcoholism is a risk factor for hepatotoxicity. However, none of the studies examining alcohol use as a risk factor for hepatotoxicity showed a significant association between the two.

Being male was found to be significantly associated with hepatotoxicity, being a protective factor rather than a risk factor. ${ }^{(21)}$ This is possibly due to the fact that androgen activity increases (induces) hepatic microsomal enzyme activity, which allows males to metabolize drugs more effectively. ${ }^{(31,34)}$ However, further studies on gender-dependent variations in drug metabolism are needed in order to draw more definitive conclusions.

With regard to genetic factors, there was a significant association between a slow NAT2 acetylator phenotype-NAT2 being the principal enzyme responsible for metabolizing isoniazidand hepatotoxicity, ${ }^{(11,18)}$ given that this phenotype can generate more hepatotoxic metabolites. ${ }^{(31,35)}$

There is a possibility of information bias as a result of combining results of studies evaluating hepatotoxicity during antituberculosis treatment, given that several studies employ no criteria for determining the severity of hepatotoxicity, which can be classified as grade $1,11,111$, or IV hepatotoxicity on the basis of transaminase levels. ${ }^{(29)}$ One limitation of the present study is that most of the reviewed studies presented no information regarding the diagnostic criteria for hepatotoxicity.

Gastrointestinal ADRs are the most common ADRs during TB treatment. ${ }^{(8)}$ They might be due to the chemical effects of the antibacterial agents, which have an effect not only on bacterial cells but also on human cells. ${ }^{(31,36)}$ They can therefore cause tissue damage in the central nervous system, peripheral nervous system, liver, and hematopoietic system. ${ }^{(37)}$ However, in the present review, most of the risk factors for gastrointestinal ADRs were not significantly associated with such reactions.

Many (37.5\%) of the reviewed studies evaluated the age of the participants; however, there were differences across studies in terms of age characterization, children and elderly individuals being evaluated. According to two studies, ${ }^{(14,15)}$ elderly patients (over 60 or 65 years of age) are more likely to have ADRs. This is due to the fact that elderly individuals have a slower metabolism, which is due to reduced enzymatic activity, reduced hepatic clearance, and reduced availability of essential endogenous cofactors. ${ }^{(31,34)}$ However, in one of the aforementioned studies, ${ }^{(14)}$ the fact that the ADRs observed in the participants were not separately categorized limits this analysis. Another limitation is related to the results of two studies. ${ }^{(21,22)}$ Although the combination of results was numerically significant in the meta-analysis, showing that being 35 years of age or older is a protective factor for hepatic ADRs in patients receiving TB treatment, it should be evaluated with caution, given that the authors' decision not to divide the participants into different population groups (e.g., elderly and non-elderly patients) is a confounding factor in the analysis of the results. The authors' decision was based on the small sample size, adults over 50 years of age constituting less than 15\% of the sample.

The studies included in the present review were conducted in the 1986-2012 period, which is an extended period of time. The major drawback of early studies (i.e., those conducted in the 1980s or in the 1990s) was their lack of methodological rigor, with little or no information regarding sample size calculation, losses to follow-up, independent variables, statistical methods, ethical issues, and study population characteristics. Another problem is that, for none of the reviewed studies, the year of publication corresponded to the year in which the study had been conducted. Among researchers who study publication bias, some consider that the time elapsed between the completion of a study and its publication is an important factor and is related to results without statistical significance. ${ }^{(10)}$

Most of the studies included in the present review were observational studies. Only 2 clinical trials were included. ${ }^{(13,19)}$ Observational studies are important because of their exploratory nature, which allows inferences to be made. However, a randomized clinical trial is the most appropriate study design to evaluate the safety profile of a given drug. ${ }^{(36,38)}$

Given that the incidence of TB is higher among poor individuals, we expected to find, among 
the studies included in the present review, at least one in which socioeconomic factors, such as income and occupation, had been evaluated. However, none of the reviewed studies examined socioeconomic variables.

The highest TB incidence rates are concentrated in African countries. However, India, China, and Indonesia together accounted for more than $40 \%$ of all TB cases in 2006. ${ }^{(39)}$ This explains why many $(44 \%)$ of the studies included in the present review were conducted in the aforementioned countries.

The relationship between TB and socioeconomic indicators appears to be associated with the level of spatial aggregation and the particular characteristics of geographic areas. ${ }^{(40)}$ In a study conducted in London, UK, it was found that for each 1\% increase in the proportion of households with more than one person per room, the average TB notification rate increased by $12 \%{ }^{(41)}$ However, the association between adverse reactions to antituberculosis drugs and socioeconomic factors has yet to be confirmed.

There is, however, an association between a low income and malnutrition, the latter being related to physiological changes. A low protein diet (poor nutrition) is related to changes in T-cell-mediated immune function, increasing susceptibility to $M$. tuberculosis infection and diseases. ${ }^{(40)}$ This explains the results of one study, ${ }^{(16)}$ in which albumin, iron, and sodium deficiency was a risk factor for fever, as was the use of alcohol.

ADRs are more closely monitored in a hospital setting, where complaints and symptoms are continuously monitored. In addition, the hospital setting allows fewer losses to follow-up and more detailed data collection in longitudinal studies. ${ }^{(13)}$ In a study conducted in the outpatient clinic of a teaching hospital in the city of São Paulo, Brazil, the frequency of minor adverse reactions was $41.1 \%$ and that of major adverse reactions was $12.8 \% .{ }^{(7)}$ According to the authors of the study, the difference between their findings and those of other studies (in which the incidence was lower) might be due to the fact that patients treated at the outpatient clinic of the medical teaching hospital are routinely screened for all possible side effects. In the present review, most of the study populations consisted of inpatients. However, in several countries, the primary treatment regimens are delivered on an outpatient basis. ${ }^{\left({ }^{(8)}\right.}$
It can be assumed that ADRs are underreported because of the difficulty in identifying such reactions and the difficulty in monitoring patients undergoing treatment. This limits the generalization of the results of the present study. Although patients are instructed to seek medical attention at a primary care clinic should any symptom arise, they rarely do in cases of mild ADRs. In addition, there is a long interval between medical visits, and ADRs are not usually reported or treated properly.

Another limitation of the present review is related to our methodological rigor; articles that did not meet all of the inclusion criteria were excluded from the review. In other reviews, rating scales are used in order to assist in the evaluation of the studies; therefore, rather than being excluded for not meeting methodological and statistical criteria, studies simply receive low ratings on these items. ${ }^{(42)}$

Although some of the risk factors analyzed in the present review were found to be significantly associated with ADRs, most of the results did not allow us to establish correlations, given that outcomes and exposure were defined differently across studies and therefore constituted an obstacle to our meta-analysis.

\section{Final considerations}

In the present review, ADRs were significantly associated with age, gender, treatment regimen, alcoholism, HIV co-infection, genetic factors, and nutritional deficiencies. Individual factors such as showing a rapid/intermediate NAT2 acetylator phenotype, being 35 years of age or older, and being male are protective factors for hepatic ADRs in patients receiving antituberculosis treatment. The remaining results should be interpreted cautiously, given that most of the data collected precluded a meta-analysis and, consequently, an evaluation of heterogeneity and external validity. The present systematic review can guide future studies aimed at achieving TB control for the benefit of public health.

\section{References}

1. Brasil. Ministério da Saúde. Secretaria de Vigilância em Saúde. Departamento de Vigilância Epidemiológica. Manual de recomendações para o controle da tuberculose no Brasil. Brasília: Ministério da Saúde; 2011.

2. Maciel EL, Guidoni LM, Favero JL, Hadad DJ, Molino LP, Jonhson JL, et al. Adverse effects of the new tuberculosis 
treatment regimen recommended by the Brazilian Ministry of Health. J Bras Pneumol. 2010;36(2):232-8.

3. Awofeso N. Anti-tuberculosis medication side-effects constitute major factor for poor adherence to tuberculosis treatment. Bull World Health Organ. 2008;86(3):B-D.

4. Hepler CD, Strand LM. Opportunities and responsibilities in pharmaceutical care. Am J Hosp Pharm. 1990;47(3):533-43.

5. Villa TC, Brunello ME, Arcêncio RA, Sassaki CM, Assis EG, Gonzalez Rl. Factors predicting unfavorable results in tuberculosis treatment: an integrative literature review (2001-2005) [Article in Portuguese]. Online Braz J Nurs [serial on the Internet]. 2007 Oct [cited 2014 May 23];7(0):[about 13 p.]. Available from: http://www. objnursing.uff.br/index.php/nursing/article/view/1098

6. World Health Organization. The importance of pharmacovigilance: safety monitoring of medicinal products. Geneva: World Health Organization; 2002.

7. Vieira DE, Gomes M. Adverse effects of tuberculosis treatment: experience at an outpatient clinic of a teaching hospital in the city of São Paulo, Brazil. J Bras Pneumol. 2008;34(12):1049-55. http://dx.doi. org/10.1590/S1806-37132008001200010

8. Brasil. Ministério da Saúde. Secretaria de Políticas de Saúde. Departamento de Atenção Básica. Manual técnico para o controle da tuberculose: cadernos de atenção básica $n^{\circ} 6$. Brasília: Ministério da Saúde; 2002.

9. Conde MB, Melo FA, Marques AM, Cardoso NC, Pinheiro VG, Dalcin Pde T, et al. Ill Brazilian Thoracic Association Guidelines on tuberculosis. J Bras Pneumol. 2009;35(10):1018-48.

10. Brasil PE, Braga JU. Meta-analysis of factors related to health services that predict treatment default by tuberculosis patients. Cad Saude Publica. 2008;24 Suppl 4:s485-502. http://dx.doi.org/10.1590/ S0102-311X2008001600003

11. Khalili H, Fouladdel S, Sistanizad M, Hajiabdolbaghi M, Azizi E. Association of $\mathrm{N}$-acetyltransferase-2 genotypes and anti-tuberculosis induced liver injury; first case-controlled study from Iran. Curr Drug Saf. 2011;6(1):17-22. http:// dx.doi.org/10.2174/157488611794479946

12. Nanashima K, Mawatari T, Tahara N, Higuchi N, Nakaura A, Inamine $\mathrm{T}$, et al. Genetic variants in antioxidant pathway: risk factors for hepatotoxicity in tuberculosis patients. Tuberculosis (Edinb). 2012;92(3):253-9. http:// dx.doi.org/10.1016/j.tube.2011.12.004

13. A controlled clinical trial of 3- and 5-month regimens in the treatment of sputum-positive pulmonary tuberculosis in South India. Tuberculosis Research Centre, Madras, and National Tuberculosis Institute, Bangalore. Am Rev Respir Dis. 1986;134(1):27-33.

14. Ai X, Men K, Guo L, Zhang T, Zhao Y, Sun X, et al. Factors associated with low cure rate of tuberculosis in remote poor areas of Shaanxi Province, China: a case control study. BMC Public Health. 2010;10:112. http:// dx.doi.org/10.1186/1471-2458-10-112

15. Baghaei P, Tabarsi P, Chitsaz E, Saleh M, Marjani M, Shemirani $\mathrm{S}$, el al. Incidence, clinical and epidemiological risk factors, and outcome of drug-induced hepatitis due to antituberculous agents in new tuberculosis cases. Am J Ther. 2010;17(1):17-22. http://dx.doi.org/10.1097/ MJT.0b013e31818f9eae

16. Barnes PF, Chan LS, Wong SF. The course of fever during treatment of pulmonary tuberculosis. Tubercle. 1987;68(4):255-60. http://dx.doi. org/10.1016/0041-3879(87)90065-1
17. Cantalice Filho JP, Bóia MN, Sant Anna CC. Analysis of the treatment of pulmonary tuberculosis in elderly patients at a university hospital in Rio de Janeiro, Brazil. J Bras Pneumol. 2007;33(6):691-8. http://dx.doi.org/10.1590/ S1806-37132007000600013

18. Cho HJ, Koh WJ, Ryu YJ, Ki CS, Nam MH, Kim JW, et al. Genetic polymorphisms of NAT2 and CYP2E1 associated with antituberculosis drug-induced hepatotoxicity in Korean patients with pulmonary tuberculosis. Tuberculosis (Edinb). 2007;87(6):551-6. http://dx.doi.org/10.1016/j. tube.2007.05.012

19. Hong Kong Chest Service/British Medical Research Council. Acceptability, compliance, and adverse reactions when isoniazid, rifampin, and pyrazinamide are given as a combined formulation or separately during three-timesweekly antituberculosis chemotherapy. Am Rev Respir Dis. 1989;140(6):1618-22. http://dx.doi.org/10.1164/ ajrccm/140.6.1618

20. Kelly PM, Cumming RG, Kaldor JM. HIV and tuberculosis in rural sub-Saharan Africa: a cohort study with two year follow-up. Trans R Soc Trop Med Hyg. 1999;93(3):28793. http://dx.doi.org/10.1016/S0035-9203(99)90025-1

21. Lee AM, Mennone JZ, Jones RC, Paul WS. Risk factors for hepatotoxicity associated with rifampin and pyrazinamide for the treatment of latent tuberculosis infection: experience from three public health tuberculosis clinics. Int J Tuberc Lung Dis. 2002;6(11):995-1000.

22. Martínez Sanchís A, Calpe Calpe JL, Llavador Ros G, Ena $\mathrm{Mu}-\mathrm{oz} \mathrm{J}$, Calpe Armero A. Primary prevention and treatment of latent tuberculosis infection with isoniazid: efficacy of a control program, 1997-2002 [Article in Spanish]. Arch Bronconeumol. 2005;41(1):27-33. http:// dx.doi.org/10.1157/13070281

23. Sharma SK, Balamurugan A, Saha PK, Pandey RM, Mehra NK. Evaluation of clinical and immunogenetic risk factors for the development of hepatotoxicity during antituberculosis treatment. Am J Respir Crit Care Med. 2002;166(7):916-9. http://dx.doi.org/10.1164/ rccm. 2108091

24. Sirinak C, Kittikraisak W, Pinjeesekikul D, Charusuntonsri P, Luanloed P, Srisuwanvilai LO, et al. Viral hepatitis and HIV-associated tuberculosis: risk factors and TB treatment outcomes in Thailand. BMC Public Health. 2008;8:245. http://dx.doi.org/10.1186/1471-2458-8-245

25. Teixeira RL, Morato RG, Cabello PH, Muniz LM, Moreira Ada S, Kritski AL, et al. Genetic polymorphisms of NAT2, CYP2E1 and GST enzymes and the occurrence of antituberculosis drug-induced hepatitis in Brazilian TB patients. Mem Inst Oswaldo Cruz. 2011;106(6):716-24. http://dx.doi.org/10.1590/S0074-02762011000600011

26. Teleman MD, Chee CB, Earnest A, Wang YT. Hepatotoxicity of tuberculosis chemotherapy under general programme conditions in Singapore. Int J Tuberc Lung Dis. 2002;6(8):699-705.

27. Klasco RK (Ed): DRUGDEX ${ }^{\circledR}$ System. [database on the Internet]. Greenwood Village (CO): Thomson Micromedex. c1974-2012 - [cited 2014 May 23]. Available from: http://www.thomsonhc.com/hcs/librarian/ND_T/HCS/ ND_PR/Main/CS/BAB6CF/DUPLICATIONSHIELDSYNC/ B80A93/ND_PG/PRIH/ND_B/HCS/SBK/3/ND_P/Main/ PFActionld/hcs.common.RetrieveDocumentCommon/ Docld/0028/ContentSetld/31/SearchTerm/isoniazid\%20/ SearchOption/BeginWith

28. Martínez Alfaro EM, Cuadra F, Solera J, Maciá MA, Geijo P, Sánchez Martínez PA, et al. Evaluation of 2 tuberculosis chemoprophylaxis regimens in patients infected with human immunodeficiency virus. The GECMEl Group 
[Article in Spanish]. Med Clin (Barc). 2000;115(5):161-5. http://dx.doi.org/10.1016/S0025-7753(00)71496-5

29. Coca MN. Hepatotoxicidade ao esquema rifampicina, isoniazida e pirazinamida no tratamento da tuberculose em pacientes com e sem a síndrome da imunodeficiência humana adquirida [dissertation]. Dissertação (mestrado). Belo Horizonte: Faculdade de Medicina da Universidade Federal de Minas Gerais; 2009.

30. Breen RA, Miller RF, Gorsuch T, Smith CJ, Schwenk A, Holmes W, et al. Adverse events and treatment interruption in tuberculosis patients with and without HIV co-infection. Thorax. 2006;61(9):791-4. http:// dx.doi.org/10.1136/thx.2006.058867

31. Katzung BG. Farmacologia básica e clínica. 10th ed. São Paulo: McGraw Hill; 2010.

32. Mincis M, Mincis R. Doença hepática alcoólica. RBM [serial on the Internet]. 2010 Jun [cited 2014 May 23];67(1):21-31. Available from: http://www.moreirajr. com.br/revistas.asp?fase $=$ r003ctid_materia $=4336$

33. Guengerich FP. Role of cytochrome P450 enzymes in drug-drug interactions. Adv Pharmacol. 1997;43:7-35. http://dx.doi.org/10.1016/S1054-3589(08)60200-8

34. Matos LC, Martins B. Hepatites tóxicas: revisão da literatura. Med Intern. 2005;4(12):239-58.

35. Possuelo LG. Estudo de polimorfismo presente no gene que codifica $\mathrm{N}$ - acetiltransferase 2 e associação com hepatotoxicidade em paciente com tuberculose tratados com RHZ [thesis]. Porto Alegre: Universidade Federal do Rio Grande do Sul; 2008.
36. Fuchs FD, Wannmacher L, Ferreira MB. Farmacologia Clínica: Fundamentos da terapêutica racional. 3rd ed. Rio de Janeiro: Guanabara Koogan; 2004.

37. Bisaglia JB, Santussi WM, Guedes AG, Gomes AP, Oliveira PC, Siqueira-Batista R. Atualização terapêutica em tuberculose: principais efeitos adversos dos fármacos. Bol. Pneumol Sanit. [serial on the Internet]. 2003 Dec [cited 2014 May 23];11(2):53-9. Available from: http://scielo. iec.pa.gov.br/scielo.php?script=sci_arttext\&tpid=S0103460X2003000200008ctlng $=p t$

38. Medronho RA. Epidemiologia. 2nd ed. São Paulo: Atheneu; 2009.

39. World Health Organization. A practical handbook on the pharmacovigilance of medicines used in the treatment of tuberculosis: enhancing the safety of the TB patient. Geneva: World Health Organization; 2012.

40. San Pedro A, Oliveira RM. Tuberculosis and socioeconomic indicators: systematic review of the literature [Article in Portuguese]. Rev Panam Salud Publica. 2013;33(4):294301. http://dx.doi.org/10.1590/S1020-49892013000400009

41. Mangtani P, Jolley DJ, Watson JM, Rodrigues LC. Socioeconomic deprivation and notification rates for tuberculosis in London during 1982-91. BMJ. 1995;310(6985):963-6. http://dx.doi.org/10.1136/ bmj.310.6985.963

42. Sampaio RF, Mancini MC. Estudos de revisão sistemática: um guia para síntese criteriosa da evidência científica. Rev Bras Fisioter. [serial on the Internet]. 2007 Feb [cited 2014 May 23];1(11):83-9. Available from: http://www.scielo.br/scielo.php?script=sci_arttextEtp id $=$ S1413-35552007000100013

\section{About the authors}

\section{Laíse Soares Oliveira Resende,}

Technical Assistant. Espírito Santo State Court of Justice, Vitória, Brazil.

\section{Edson Theodoro dos Santos-Neto}

Professor. Federal University of Espírito Santo, Vitória, Brazil. 